\title{
Tobacco Smoke Activates Protein Association and Tyrosine Phosphoryla- tion of the GPI-Transamidase Complex Subunits in Human Cancers
}

\author{
Yiping Huang ${ }^{\#, 1}$, Jatin Nagpal ${ }^{\#, 2}$, Barry Trink ${ }^{2}$, and Edward Ratovitski*,1,2 \\ ${ }^{I}$ Departments of Dermatology, ${ }^{2}$ Otolaryngology/Head and Neck Surgery, The Johns Hopkins University School of \\ Medicine, Baltimore, Maryland 21231, USA
}

\begin{abstract}
We previously found that the protein subunits of GPI-transamidase complex (e.g. PIG-U, PIG-T and GPAA1) play critical roles of oncogenes in human bladder and breast cancers manifesting their activities through signaling mechanisms that involve urokinase receptor/Stat3 and paxillin pathways. We report here that cigarette smoke extract (CSE) enhanced colony formation and invasiveness of certain human cancer cells (e.g. head and neck, bladder or breast). We found that CSE induced the complex formation between PIG-U, PIG-T and GPAA1 proteins and also the association between GPAA1 and EGFR in cancer cells. We observed that inhibitors of EGFR tyrosine kinase, Gefitinib or Erlotinib, modulated tyrosine phosphorylation of PIG-U and PIG-T induced by CSE. We also found that EGFR tyrosine kinase inhibitors and siRNA silencing of PIG-U, PIG-T or GPAA1 modulated the CSE-induced increase in colony formation and invasiveness of human bladder cancer cells. We suggest that the novel mechanism overlapping the oncogenic potential of PIG-U, PIG-T and GPAA1 implicates EGFR tyrosine phosphorylation of PIG-U or PIG-T and subsequent paxillin phosphorylation.
\end{abstract}

\section{INTRODUCTION}

The worldwide research has produced findings showing side stream smoke second-hand smoking or involuntary smoking) to be harmful and possibly deadly to nonsmokers [1-3]. The report sponsored by the Environmental Protection Agency concluded that second-hand cigarette smoke is a major cause of indoor air pollution [2-7]. Second-hand smoking is responsible for deaths of more than 7,000 infants and 53,000 non-smoking adults annually [2-7]. Carcinogenesis of head and neck, breast and bladder in humans is greatly induced from field-wide tobacco exposure of the upper aerodigestive tract (oral cavity, larynx, esophagus, lung, stomach) generated from active tobacco smoking and second-hand smoking [4, 8-22]. The incidence of these cancers is increasing in both men and women, and active smoking/secondhand smoking is the most important factor [4, 9-22]. Moreover, studies show that second-hand smoking is much more critical in initiation or promotion of head and neck, breast and bladder cancers than genetic factors [23-25].

Recently, our research team has discovered a novel oncogene, CDC91L1 (PIG-U), which was associated with human bladder cancer [26-28]. The PIG-U protein was first found to be an integral part of membrane-associated glycosylphosphatidyl inositol (GPI)-anchoring complex, which modified numerous proteins (e.g. receptors, etc.) by GPItransamidase enzymatic reaction by attaching the GPIanchor to newly synthesized proteins and then targeting them into membranal microenvironment [29-32]. The mammalian GPI transamidase is a protein complex of at least five subunits, GPI8, GPAA1, PIG-S, PIG-T and PIG-U [31, 32].

*Address correspondence to this author at the Cancer Research Building-2, Rm 2M05, 1550 Orleans Street, Baltimore, MD 21231, USA;

E-mail: eratovi1@jhmi.edu

"These authors contributed equally to this work.
Some of us have previously found that CDC91L1 was amplified/overexpressed in $>75 \%$ of bladder cancer cell lines and primary tumors [26, 27]. Then we further found that GPAA1 is amplified/overexpressed in head and neck cancer cells and both GPAA1 and PIG-T are amplified/overexpressed in breast cancer cells [27, 28]. We also found that the forced overexpression of PIG-U malignantly transformed NIH3T3 cells in vitro and in vivo and also resulted in up regulation of the urokinase receptor, a GPIanchored protein, and in turn increased STAT3 phosphorylation in bladder cancer cells [26].

We hypothesized that exposure human cancer cells to cigarette smoke exposure (extract, CSE) will trigger the signal transduction pathway implicating the GPI-anchoring complex leading to an increase in cell invasiveness and migration. We suggested that the oncogenic function of PIG-U is manifested through its interaction with both soluble and membrane-associated proteins. We, therefore, found that PIG-U forms protein complexes with several protein candidates (e.g. PIG-T, GPAA1, paxillin, EGFR) playing a critical role in tumorigenesis, cell adhesion and apoptosis upon cigarette smoke exposure. Our findings implicated the GPI anchoring system as a potential oncogenic pathway and therapeutic target in human cancers upon cigarette smoke exposure. We think that further understanding of biological function of PIG-U and other members of GPI-anchoring complex (PIG-T or GPAA1) provide us with critical tools to design chemotherapeutic interventions beneficial for patients with bladder, breast and head and neck cancers.

\section{MATERIALS AND METHODOLOGY}

\section{Cells, Reagents and Antibodies}

We used human embryonic kidney cells (HEK293, CRL1573,), human bladder transitional cell carcinoma cells (J82, HTB-1), human breast adenocarcinoma cells (MCF7, HTB- 
22). All these cell lines were obtained from the American Tissue Culture Collection (ATCC) and maintained in the Eagle's Minimum Essential Medium and 10\% fetal bovine serum (FBS). We also used head and neck squamous cell carcinoma (HNSCC) cell line 029 (expressing wild type p53 and p63) was isolated at the Department of Otolaryngology/Head and Neck Surgery of the Johns Hopkins University School of Medicine. 029 cells were maintained in RPMI medium 1640, 10\% FBS. Cell viability and growth was monitored by MTT kit (ATCC).

Epidermal growth factor (EGF) was purchased from Biosource International Inc. Gefitinib (GEF, Iressa, AstraZeneca) was dissolved in DMSO $(10 \% \mathrm{v} / \mathrm{v}$ final concentration) and diluted in carboxymethylcellulose $(0.25 \% \mathrm{w} / \mathrm{v})$ to $20 \mathrm{mg} / \mathrm{ml}$. Erlotinib (ERLO, Tarceva) was generously provided by OSI Pharmaceuticals. Stock solutions of Erlotinib ( $5 \mathrm{mmol} /$ liter) were prepared in demethylsulfoxide (DMSO) and stored in aliquots at $-20^{\circ} \mathrm{C}$. The maximum dose of Erlotinib for the studies reported here was $20 \mu \mathrm{mol} /$ liter due to drug precipitation at higher concentrations.

Cells were treated with cigarette smoke extract (CSE), which was prepared as follows: one 100-mm cigarette without filter (Research Grade Cigarette, University of Kentucky) will be combusted with a Variable Speed Pump (Fisher Scientific) through $50 \mathrm{ml}$ 1xphosphate buffer saline at a rate of $500 \mathrm{cc} / \mathrm{min}$. The resulting suspension $(100 \%$ aqueous CSE) will be filtered through $0.22 \mu \mathrm{M}$ filter and used in cell culture experiments [11, 12, 16, 22, 25, 33].

We used a rabbit polyclonal antibody against human paxillin (\#RB-10643-P0) purchased from Lab Vision, a mouse monoclonal antibody against human paxillin (clone 5H11, \#ab3127) and a mouse monoclonal anti-phospho-tyrosine (anti-PY) antibody (\#ab45287) both obtained from Abcam and a rabbit polyclonal antibody against tyrosinephosphorylated human paxillin (p-paxillin-Y-31, sc-14035R) was obtained from Santa Cruz Biotechnology. The horseradish peroxidase-conjugated secondary antibodies (antimouse, anti-rabbit or anti-goat immunoglobulins (GE Healthcare Bio-Sciences Corp.) were used for immunoblotting and immunoprecipitation as previously described [26, $28,34]$. We also used a mouse polyclonal antibody against human GPAA1 (\#H00008733-A01, Abnova/Novus Biologicals), a rabbit polyclonal antibody against human GPAA1 (\#10104-1-AP) from Proteintech Group Inc, a mouse monoclonal antibody against PIG-T (Orbigen), and a custom rabbit polyclonal antibody against human PIG-U (EVVSPLSSWKRVVEGL) was obtained from Alpha Diagnostic.

\section{Yeast 2-Hybrid Screening}

A yeast 2-hybrid screen was performed using the Matchmaker Gal4 system 3 with 2-hybrid cDNA libraries from normal human keratinocytes, HeLa, fetal human kidney and fetal human brain (all from Stratagene) as described elsewhere [34]. All clones were verified for their ability to grow on different selective media, SD/Trp-Leu-, SD/HisTrp-Leu-, and/or SD/Ade-His-Trp-Leu- in the presence of Xgal to screen for ADE2, HIS3 and MEL1 expression. As positive and negative controls, we used yeast expression constructs for p53/SV40 pair (positive control) or SV40/lamin C pair, respectively (negative control). In addi- tion, the intermolecular interactions between bait and prey proteins were verified by a subsequent transformation of both plasmids into the AH109 yeast strain followed by $\alpha$ and $\beta$-galactosidase assays [34].

\section{Immunoblotting and Immunoprecipitation}

Cells were lysed in a buffer A (50 mM Tris, $\mathrm{pH} 7.5,100$ $\mathrm{mM} \mathrm{NaCl}, 2 \mathrm{mM}$ EDTA, $0.5 \%$ Triton X-100, 0.5\% Brij-50, 1 mM PMSF, $0.5 \mathrm{mM} \mathrm{NaF}, 0.1 \mathrm{mM} \mathrm{Na} \mathrm{VO}_{4}, 2 \mathrm{X}$ complete protease inhibitor cocktail), sonicated five times for $10 \mathrm{sec}$ time intervals, and clarified for $30 \mathrm{~min}$ at 15,000xg (Huang et al., 2008a, b). Supernatants (designated as total lysates) were resolved by $4-10 \%$ SDS-PAGE and then analyzed by immunoblotting or immunoprecipitation, as previously described [28, 34].

\section{Expression Vectors and Transfection}

Full length PIG-U, PIG-T and GPAA1 open reading frame cDNA sequences [28] were amplified by PCR with primers containing the $1 \mathrm{x}$ HA sequence at the 3 '-end and cloned into the pIRES-EGFP expression vector (Invitrogen). Cells were transfected with $1 \mu \mathrm{g}$ of an empty pIRES-EGFP vector or with the PIG-U/PIG-T/GPAA1 expression constructs using FuGENE 6 transfection reagents (Roche Molecular Diagnostics) in 6 well plates according to the manufacturer's transfection protocol and as described elsewhere $[28,34]$.

\section{Small RNA Interference (SiRNA)}

SiRNA constructs were designed using the GeneSuppressor System. We designed two siRNA constructs for GPAA1 and PIG-U and three siRNA constructs each for PIG-T as previously described [26, 28]. For the negative control plasmid, a scrambled sequence is provided in the GeneSuppressor manual from Imgenex as described [26, 28].

\section{Colony Formation Assay}

Cell survival was determined by plating 5 to $9 \times 10^{3}$ cells in 60-mm dishes. After $24 \mathrm{~h}$, various concentrations of Gefitinib or Erlotinib were added, followed by incubation for 10 14 days at $37^{\circ} \mathrm{C}$. Both Gefitinib and Erlotinib were solubilized in DMSO. For control experiments the equivalent volumes of DMSO were added to plates. Cells were placed in 1 $\mathrm{ml}$ of $0.3 \%$ low melting agarose in $10 \%$ fetal bovine serum (FBS)/MEM (MCF7 or J82 cells) or $10 \%$ FBS/RPMI (029 cells) media and then overlaid onto $1 \mathrm{ml}$ of $0.5 \%$ agarose/10\% FBS/DMEM/RPMI in each well of a 6-well plate. The medium was changed every 3 days and the clones were allowed to grow for 10-14 days. Colony formation (2-3mm) was counted after Giemsa staining and each experiment was repeated in triplicate [26, 28].

\section{Cell Invasion Assay}

Cell invasion was quantified using a Cell Invasion Colorimetric Assay Kit, QCM ${ }^{\mathrm{TM}}$ (ECM550, Millipore Corporation) or with Matrigel invasion chamber assay (BD Biosciences) according to the manufacturer's instructions and as previously described [28]. Cells $\left(10^{5}\right)$ were seeded in each well of a 24-well plate. The cells were transfected with siRNA and then invasive cells were counted as previously described [26, 28]. Statistical analysis of three independent experiments was done using Student $t$ test [26, 28]. Data are expressed as the mean \pm S.D. 


\section{RESULTS}

Some of us have previously shown that the GPI-complex members (PIG-U, GPAA1 and PIG-T) are amplified and/or overexpressed in bladder, breast and head and neck cancers compared to control normal tissue counterparts [26-28, 35].

Here, we analyzed the effect of cigarette smoke extract (CSE) on the protein levels of the GPI-transamidase complex subunits (e.g. PIG-U, PIG-T and GPAA1) in bladder, breast and head/neck cancer cell lines, respectively. We observed that CSE (1-8\%) gradually induced protein levels of PIG-U, PIG-T, GPAA1 in bladder cancer, breast cancer and head/neck squamous cell carcinoma cells, respectively (Fig. 1).

\section{Bladder (J82) Con 1\% 2\% 5\% 8\%}

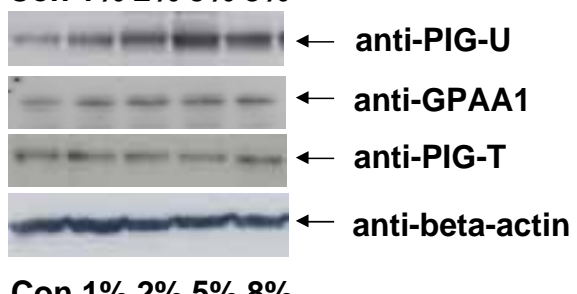

\section{Breast (MSF7) Con 1\% 2\% 5\% 8\%}

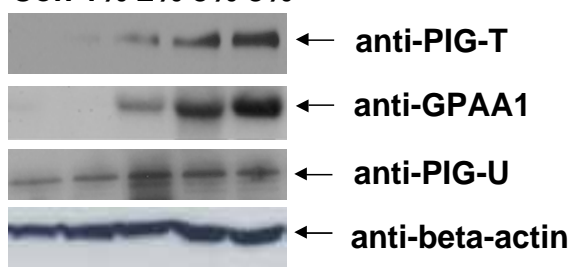

HNSCC (029) Con $1 \% 2 \% 5 \% 8 \%$

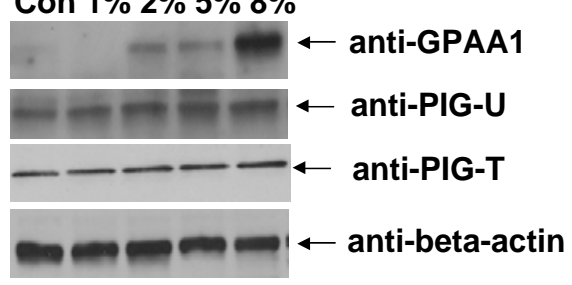

Fig. (1). CSE induces the expression of GPI-transamidase complex members (PIG-U, PIG-T and GPAA1) in human cancer cells in vitro. Cells were treated with CSE at $1 \%, 2 \%, 5 \%$ and $8 \%(\mathrm{v} / \mathrm{v})$ concentrations for 3 days followed by immunoblotting analysis with indicated antibodies. CSE-treated cells also bigger in size and were more aggregated as compared to control cells. Con - no CSE added.

We further found that PIG-U, PIG-T and GPAA1 formed protein-protein complexes with each other, while were forcefully expressed in HEK293 cells (Fig. 2A) or endogenously expressed in bladder, breast and head and neck cancer cells (Fig. 2B). Then, we observed that CSE (0.5-5\%) increased levels of the protein-protein complexes between PIG-U and GPAA1 (Fig. 2C) and between PIG-T and PIG-U proteins (data not shown) in human cancer cell lines $(029, \mathrm{~J} 82$ and MCF7).

$\mathrm{We}$, therefore, propose that the physiologic and pathophysiologic function of the GPI-transamidase complex (PIGU/PIG-T/GPAA1) might be manifested through its interaction with other cellular proteins. Using 2-hybrid screens, we found that PIG-U, PIG-T and GPAA1 associated with a
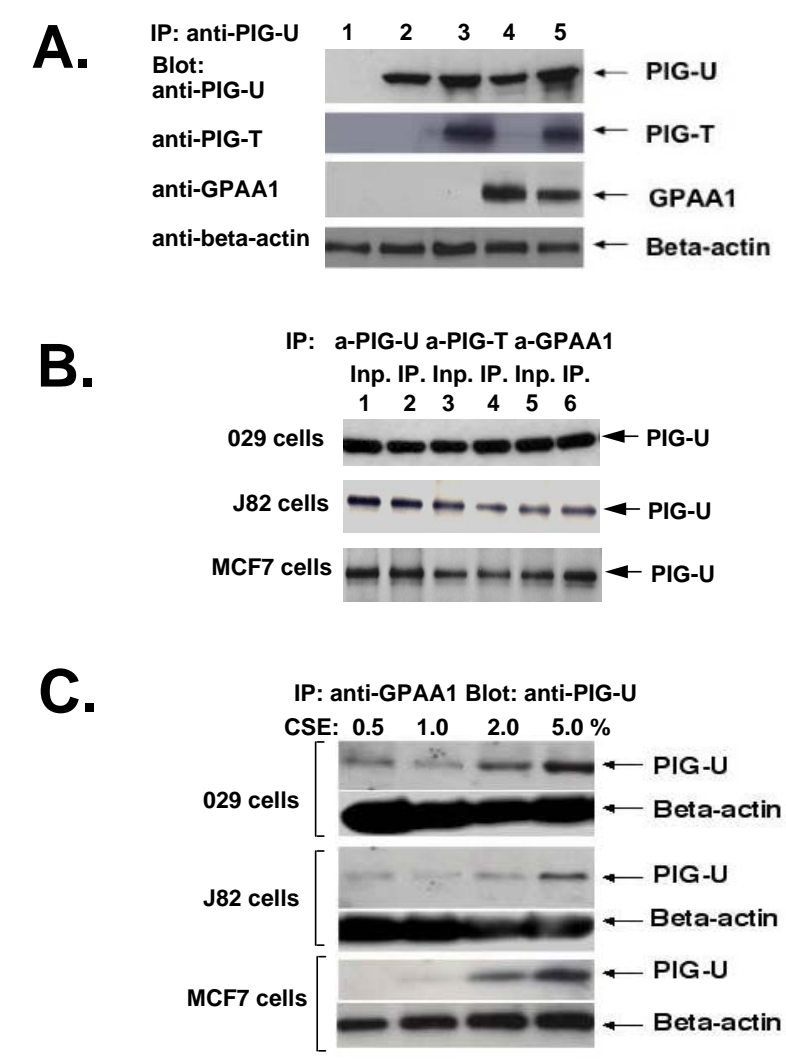

Fig. (2). CSE enhances the protein complex formation between PIG-U, PIG-T and GPAA1 in tumor cells. (A). PIG-U/PIGT/GPAA1 protein complexes in HEK293 cells. Cells were transfected with an empty vector (lane 1); the expression cassettes for PIG-U (lanes 2-5), for PIG-T (lanes 3 and 5), and for GPAA1 (lanes 4 and 5). Total lysates $(500 \mu \mathrm{l})$ were blotted with PIG-U antibody for expression levels (upper panel) and then were immunoprecipitated (IP) with the custom antibody to PIG-U and subsequently blotted with the antibodies against PIG-T or GPAA1 (two center panels). Equal amounts of total lysates used for IP were tested with anti-beta-actin antibody (lower panel). (B). Human cancer cells (029, J82 and MCF7) showed endogenous PIG-U/PIGT/GPAA1 protein complexes. Lanes 1, 3 and 5 - inputs; lanes 2, 4 and 6 - IPs with antibodies to PIG-U, PIG-T and GPAA1, respectively. immunoblotting with antibody to PIG-U. (C). Exposure to CSE (0.5-5.0\%) for 3 days gradually increased levels of the PIGU//GPAA1 protein complexes in human cancer cell lines (029, J82 and MCF7). IP was performed with an antibody to GPAA1 and immunoblotting was done with antibody against PIG-U. Equal amounts of total lysates used for IP were tested with anti-beta-actin antibody.

number of protein candidates in yeast genetic assay (Fig. 3). We generated yeast expression constructs harboring the $\mathrm{N}$ terminal half of the bait proteins (PIG-U- $\Delta$ C, residues 1-366; PIG-T- $\Delta \mathrm{C}$, residues; $1-374$ or GPAA1- $\Delta \mathrm{C}$, residues 1-412). All sequences were subcloned in frame into the Gal4 DNA binding domain $(\mathrm{BD})$ and the resulting bait plasmids were designated as pGal4-BD-PIG-U- $\Delta \mathrm{C}$, pGal4-BD- PIG-T- $\Delta \mathrm{C}$, and pGal4-BD- GPAA1- $\Delta$ C, respectively. We screened three 2-hybrid cDNA libraries from human fetal kidney, human fetal liver and human keratinocytes. Screening a total of 4.3 
x $10^{6}$ yeast transformants yielded twenty-seven positive clones for twelve individual genes representing both soluble and membrane-bound proteins (Fig. 3). The protein association between GPAA1 and EGFR attracted our special attention.

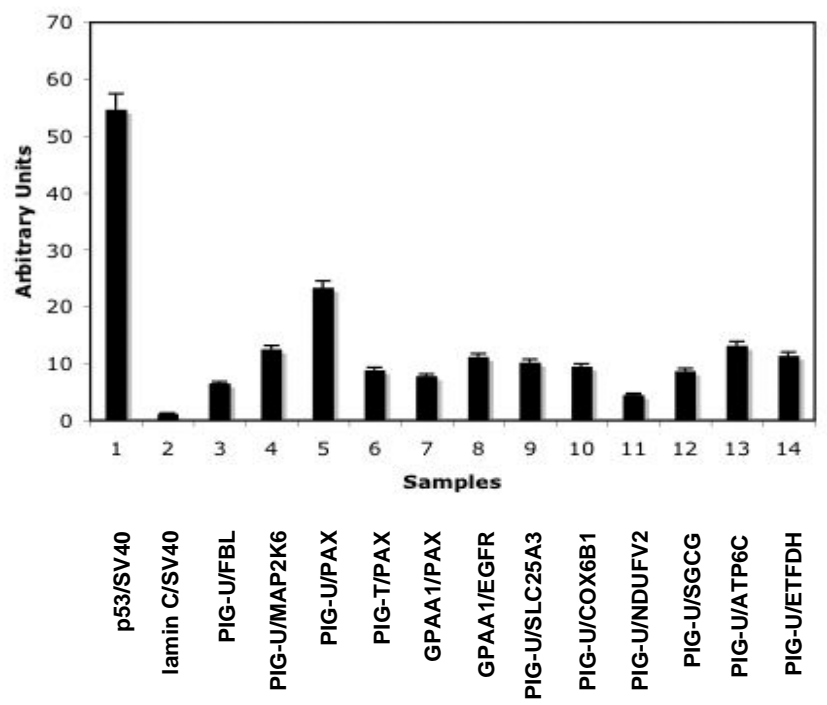

Fig. (3). Selected 2-hybrid protein candidates interacting with PIGU, PIG-T and GPAA1. Samples: 1- p53/SV40; 2- lamin C/SV40; 3PIG-U/fibrillarin (FBL); 4- PIG-U/MAP2K6; 5- PIG-U/paxillin (PAX); 6- PIG-T/PAX; 7- GPAA1/PAX; 8- GPAA1/EGFR; 9PIG-U/solute carrier protein family 25 member 3 (SLC25A3); 10PIG-U/cytochrome c oxydase subunit $6 \mathrm{~b}$, polypeptide 1 (COX6B1); 11- PIG-U/NADH dehydrogenase flavoprotein 2 (NDUFV2); 12PIG-U/sarcoglycan gamma (SGCG); 13- PIG-U/ATP-ase H (ATP6C); 14- PIG-U/electron transfer flavoprotein dehydrogenase (ETFDH). The intermolecular interactions between bait and prey proteins were verified by a subsequent transformation of both plasmids into the AH109 yeast strain followed by $\alpha$ - and $\beta$ galactosidase assays [34].

As we previously showed, the oncogenic potential of PIG-U, PIG-T and GPAA1 are associated with the phosphorylation of paxillin (For a review see refs. 5, 7, 12, 15, $34,35,26,28)$. We therefore suggested that the phosphorylation of PIG-U, PIG-T or GPAA1 themselves could contribute into regulation of their biological/oncogenic function. Our initial bioinformatics analysis using a http://wwww.phospho.elm.eu.org/scansite motif scanner) showed that both PIG-U and PIG-T contained two potential tyrosine phosphorylation sites each (PIG-U, residues 220236, 331-345; PIG-T, 274-285, 301-312) for receptor tyrosine kinase (Table 1). Whereas, GPAA1 showed only a single potential tyrosine phosphorylation site (residues 411416). These proteins also display a few putative serine/threonine phosphorylation sites (e.g. PKC-E/PRKD1 or Akt kinases, Table 1).

We next showed that the endogenous GPAA1 expressed in MCF7 breast cancer cells strikingly co-immunoprecipitated with EGFR upon EGF exposure (Fig. 4A, upper panel, lanes 5 and 6), whereas both PIG-U and PIG-T failed to form such protein complexes (Fig. 4A, upper panel, lanes 1-4). However, antibodies to PIG-U (lanes 1 and 2), PIG-T (lanes 3 and 4) or GPAA1 (lanes 5 and 6) effectively precipi-
Table 1. Phosphorylation/Protein Kinase Motifs in the PIGU, PIG-T and GPAA1

\begin{tabular}{|c|c|c|c|}
\hline Protein Name & PKC/PRKD & Tyrosine Kinase & Akt Kinase \\
\hline \hline PIG-U & $\begin{array}{c}16-32 \text { aa } \\
211-227 \text { aa }\end{array}$ & $\begin{array}{c}220-236 \text { aa } \\
331-345 \text { aa }\end{array}$ \\
\hline PIG-T & $251-260$ aa & $\begin{array}{c}274-285 \text { aa } \\
301-312 ~ a a\end{array}$ & $\begin{array}{c}52-68 \text { aa } \\
261-270 \text { aa } \\
\end{array}$ \\
& & $411-416$ aa & $364-408$ aa \\
\hline GPAA1 & $220-236$ aa & aa \\
\hline
\end{tabular}

\section{A.}

EGF: $010 \mathrm{~min} .010 \mathrm{~min} .010 \mathrm{~min}$. $\begin{array}{lcl}\text { IP: } \text { anti-PIG-U } & \text { anti-PIG-T } & \text { anti-GPAA1 } \\ \text { Blot: anti-EGFR } & \text { anti-EGFR } & \text { anti-EGFR } \\ 1 & 3 & 4\end{array}$

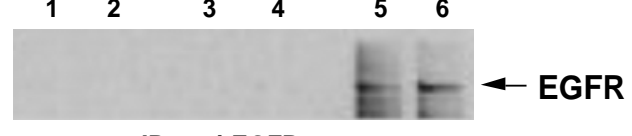

IP: anti-EGFR

Blot: anti-GPAA1 anti-PIG-T anti-PIG-U

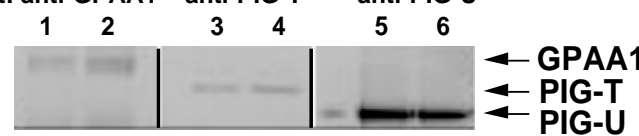

B.

EGF: $\quad 010 \mathrm{~min} .010 \mathrm{~min} .010 \mathrm{~min}$. Blot: anti-PY

IP: anti-PIG-T anti-PIG-U anti-GPAA1

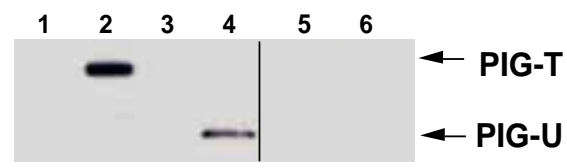

Fig. (4). EGF induces a protein interaction of GPAA1 and EGFR, while stimulates a tyrosine phosphorylation of PIG-U and PIG-T. (A). MCF7 breast cancer cells were treated with EGF $(50 \mathrm{nM})$ for 0 (lanes 1, 3 and 5) and 10 min (lanes 2, 4 and 6) and total lysates were immunoprecipitated (IP) with indicated antibodies against PIG-U (lanes 1 and 2), PIG-T lanes 3 and 4) or GPAA1 (lanes 5 and 6) shown on the upper panel and immunoblotted with an antibody to EGFR. Immunoprecipitates (IP) obtained with an antibody against EGFR (shown on the lower panel) were detected with antibodies against GPAA1 (lanes 1 and 2), PIG-T (lanes 3 and 4) and PIG-U (lanes 5 and 6). Vertical lines separate independent experiments. (B). MCF7 breast cancer cells were exposed to $50 \mathrm{nM}$ EGF for 0 (lanes 1, 3 and 5) and 10 min (lanes 2, 4 and 6). Total lysates were precipitated with an antibody to PIG-T (lanes 1 and 2) or an antibody to PIG-U (lanes 2 and 4) or antibody to GPAA1 (lanes 5 and 6) and blotted with anti-phosphotyrosine antibody (anti-PY).

tated the corresponding members of GPI-complex (Fig. 4A, lower panel). At the same time, while both PIG-U and PIG-T were phosphorylated upon EGF exposure, GPAA1 failed to show such protein modification after EGF treatment (Fig, 4B, lanes 2 and 4).

We then exposed human cancer cells (bladder, breast or head and neck) to slightly lower doses of CSE (0.2-1.0\%). We found that CSE (1\%) induced tyrosine phosphorylation of PIG-U and PIG-T (Fig. 5A), while also induced a protein 
association between GPAA1 and EGFR (Fig. 5B). Thus, we suggest that either EGF or CSE modulated the complex formation between EGFR and GPAA1 in turn tethering PIG-U or PIG-T for the tyrosine phosphorylation by EGFR tyrosine kinase.
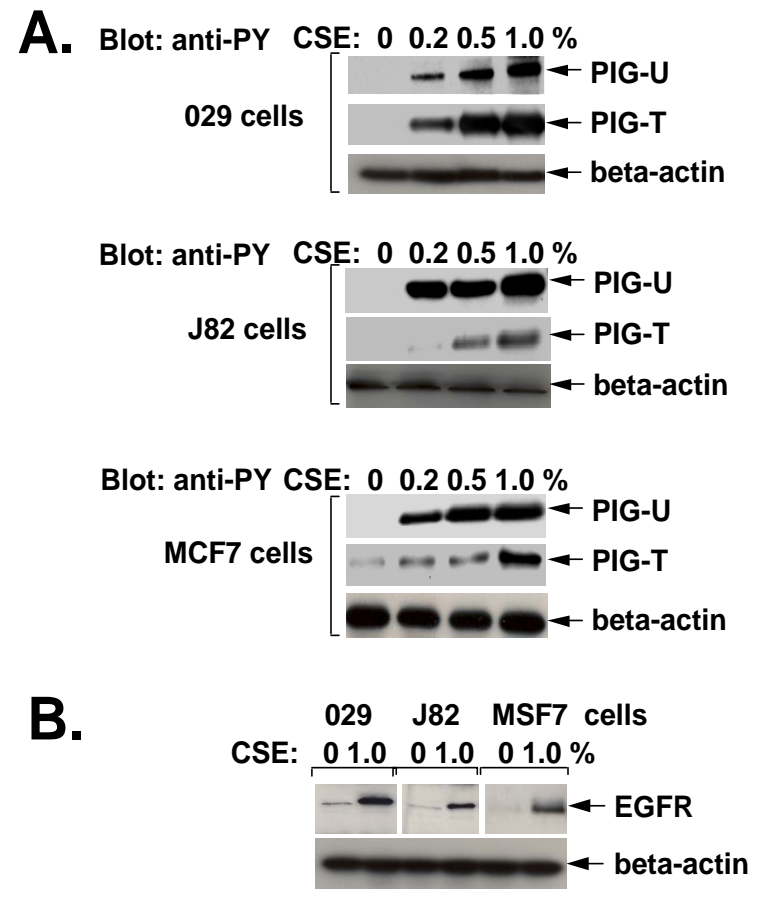

Fig. (5). CSE induces tyrosine phosphorylation of PIG-U and PIG$\mathrm{T}$, while stimulates a protein-protein complex formation between EGFR and GPAA1 in human cancer cells. (A). Cells (029, J82 or MCF7) were exposed to CSE (0-1.0\%) for 3 days and total lysates were prepared in the presence of protease and phosphatase inhibitors [26, 28, 34]. Lysates were immunoprecipitated (IP) with antibodies against PIG-U or PIG-T and subsequently immunoblotted with an antibody to phosphotyrosine (anti-PY). Protein levels of lysates used for IP were normalized by immunoblotting with an antibody against beta-actin. (B). Total lysates from 029, J82 or MCF7 cells exposed to 0 and $1.0 \%$ CSE were immunoprecipitated (IP) with an antibody against GPAA1 and then protein complexes were blotted with an antibody to EGFR. Protein levels of lysates used for IP were normalized by immunoblotting with an antibody against beta-actin. Vertical lines separate independent experiments.

To examine this hypothesis, we forcefully expressed PIG-U, PIG-T and GPAA1 in HEK293 cells and then cells were treated with the EGF or CSE along with the inhibitors for EGFR tyrosine kinase, Gefitinib (GEF, Iressa) or Erlotinib (ERLO, Tarceva). We showed that both EGFR tyrosine kinase inhibitors decreased phosphorylation levels of PIG-U and PIG-T (Fig. 6A and 6B), while failed to affect protein interaction between EGFR and GPAA1 induced by both EGF and CSE (Fig. 7A and 7B).

We then employed both the anchorage-independent cell growth assay and cell invasiveness assay to examine whether CSE and EGFR tyrosine kinase inhibitors affect a neoplastic growth of J82 human bladder cancer cells. We first found that CSE dramatically increased (by $38.6 \pm 4.5 \%$ ) a number

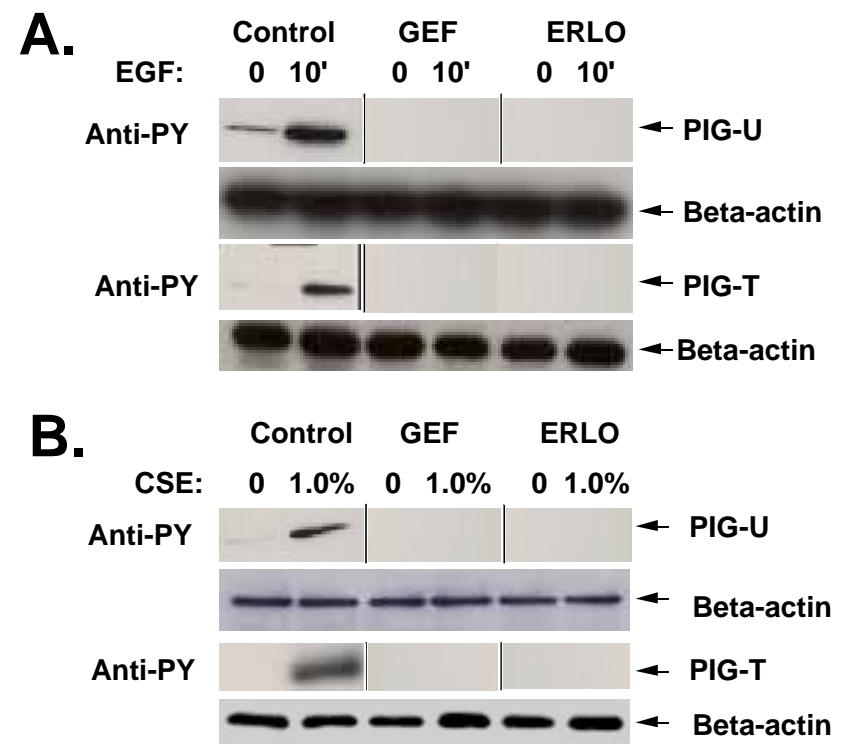

Fig. (6). EGFR tyrosine kinase inhibitors modulate tyrosine phosphorylation of PIG-U and PIG-U. (A). HEK293 cells were treated with $50 \mathrm{nM}$ EGF for 0 or $10 \mathrm{~min}(\mathbf{B})$. HEK293 cells were treated with 0 or $1.0 \%$ CSE for 3 days. Total lysates were collected after cells were additionally treated with $2 \mu \mathrm{M}$ of Gefitinib (GEF) or 5 $\mu \mathrm{M}$ of Erlotinib (ERLO) for $24 \mathrm{~h}$. Lysates were prepared in the presence of protease and phosphatase inhibitors [26, 28, 34]. Lysates were immunoprecipitated (IP) with an antibody to PIG-U or an antibody to PIG-U with a subsequent immunoblotting with an anti-phosphotyrosine antibody (anti-PY). Protein levels of lysates used for IP were normalized by immunoblotting with an antibody against beta-actin. Vertical lines separate independent experiments.

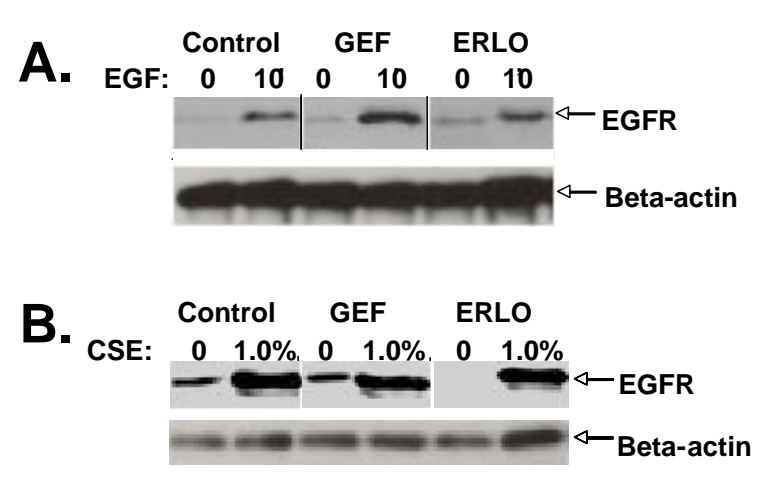

Fig. (7). EGFR tyrosine kinase inhibitors failed to affect association between GPAA1 and EGFR in HEK293 cells. (A). HEK293 cells were treated with $50 \mathrm{nM}$ EGF for 0 or $10 \mathrm{~min}$ (B). HEK293 cells were treated with 0 or $1.0 \%$ CSE for 3 days. Total lysates were collected after cells were additionally treated with $2 \mu \mathrm{M}$ of Gefitinib (GEF) or $5 \mu \mathrm{M}$ of Erlotinib (ERLO) for $24 \mathrm{~h}$. Lysates were prepared in the presence of protease and phosphatase inhibitors [26, 28, 34]. Lysates were immunoprecipitated (IP) with an antibody to GPAA1 and then blotted with an antibody to EGFR. Protein levels of lysates used for IP were normalized by immunoblotting with an antibody against beta-actin. Vertical lines separate independent experiments.

of tumor cells showing the anchorage-independent colony growth in soft agar (Fig. 8A, sample 2 vs. 1) and invasive- 
ness (Fig. 8B, sample 2 vs. 1). Next, we used siRNA to effectively knockdown the expression of PIG-U, PIG-T and GPAA1 in J82 human bladder cancer cells (Fig. 9B). J82 cells transfected with indicated siRNA were exposed to 5\% CSE and later tested for colony growth and cell invasiveness (Fig. 8A and 8B, samples 3-5 vs. sample 2). We observed that siRNA knockdown of PIG-U (sample 3, by $58.3 \pm 4.1$ $\%$ ), PIG-T (sample 4 , by $57.2 \pm 5.4 \%$ ) or GPAA1 (sample 5 , by $56.9 \pm 5.8 \%$ ) modulated the CSE effect (Fig. 8A and $\mathbf{8 B}$ ). We further observed that both Gefitinib (GEF, sample 6, by $49.8+3.8 \%$ ) and Erlotinib (ERLO, sample 7, by $52.1 \pm 4.2 \%$ ) inhibited the effect of CSE on J82 bladder cancer cell colony growth in soft agar suggesting that the EGFR tyrosine kinase plays a critical role in this process (Fig. 8A and $\mathbf{8 B}$ ). The modulatory effect of siRNA against the GPI-transamidase members or EGFR tyrosine kinase inhibitors on cell inva-
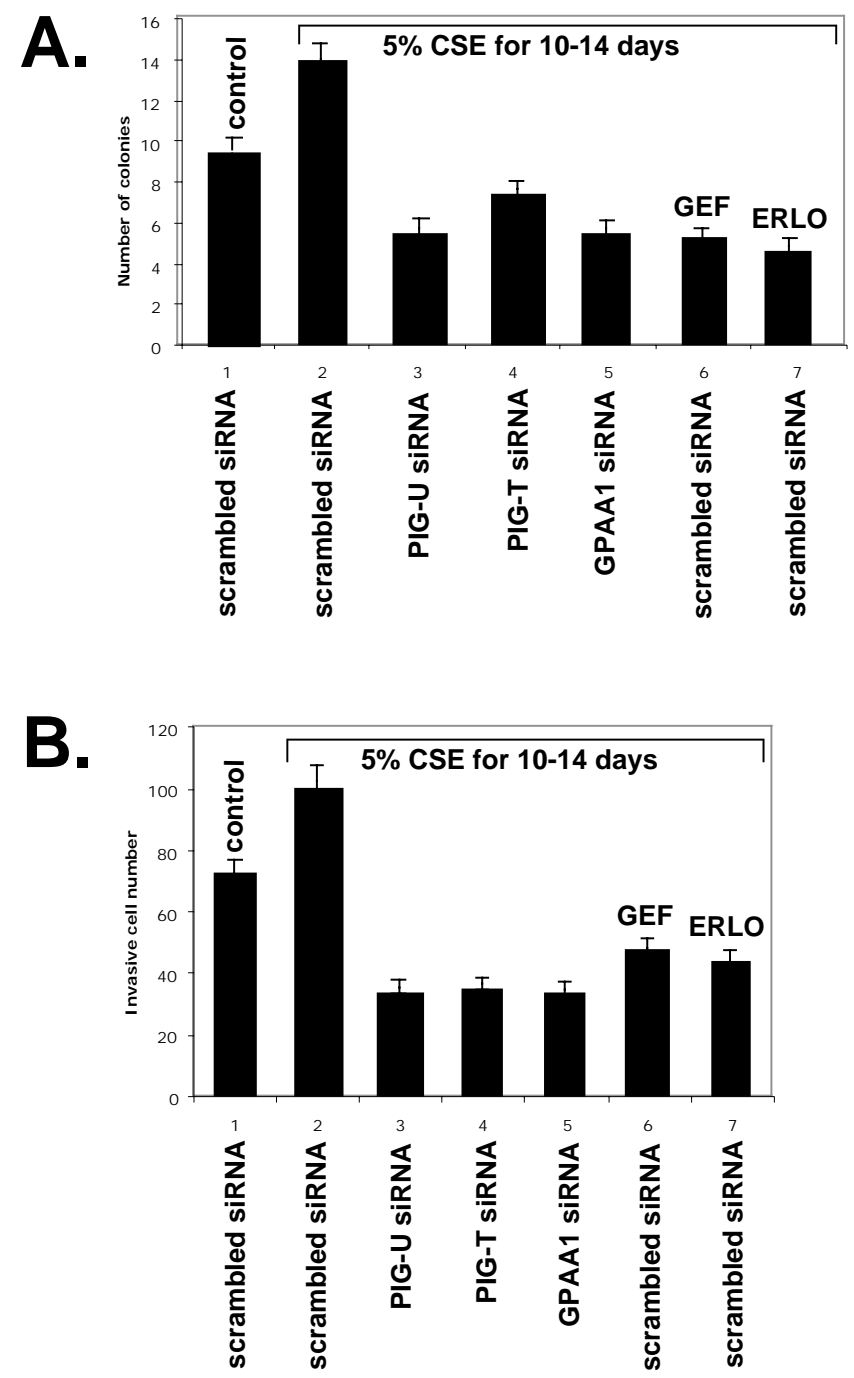

Fig. (8). CSE enhances the colony formation and invasiveness of human bladder cancer cells in vitro. (A). Colony formation assay. (B) Cell invasiveness assay. J82 bladder cancer cells were transfected with the scrambled control siRNA (samples 1, 2, 6 and 7), siRNA against PIG-U (sample 3), PIG-T (sample 4) or GPAA1 (sample 5) were exposed to control media (sample 1) or CSE (5\%, samples 2-7) for 10-14 days. Cells were also treated with $1 \mu \mathrm{M}$ of Gefitinib (GEF, sample 6) or $2 \mu \mathrm{M}$ of Erlotinib (ERLO, sample 7) for 10 days. siveness/tumor growth unequivocally implicated the PIGU/PIG-T/GPAA1/EGFR signaling pathway into these cellular processes. Similar results were obtained with 029 HNSCC cells and MCF7 breast cancer cells (data not shown).

Paxillin is well known to play a key role in tumorigenesis, cell invasiveness and cell migration [28, 36-42, 43] and we showed that paxillin promotes an oncogenic transformation in bladder and breast cancers through a PIG-U/PIGT/GPAA1-dependent mechanism [26-28]. We further tested whether CSE and EGFR inhibitors affect the tyrosine phosphorylation of paxillin in J82 human bladder cancer cells. We found that $1 \%$ CSE greatly increased the level of phosphorylated paxillin, while EGFR tyrosine kinase inhibitors (Gefitinib, GEF or Erlotininb, ERLO) decreased the levels of phosphorylation of paxillin (Fig. 9A). Similar results were obtained with 029 HNSCC and MCF7 breast cancer cells (data not shown). Interestingly, that in contrast to control (scrambled) siRNA, siRNA against PIG-U, PIG-T and GPAA1 effectively blocked the tyrosine phosphorylation of paxillin, while failed to affect the paxillin protein levels (Fig. 9B).

\section{A. Control GEF ERLO CSE: $0 \quad 1.0 \% 0 \quad 1.0 \% 0 \quad 1.0 \%$}
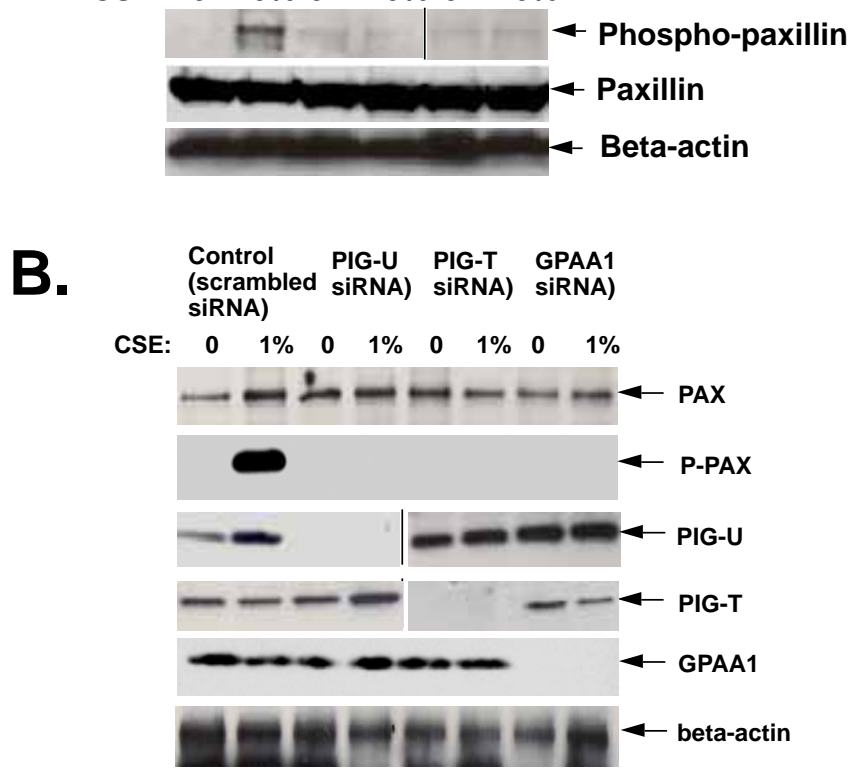

Fig. (9). CSE induces the tyrosine phosphorylation of paxillin in human bladder cancer cells. (A). J82 bladder cancer cells were treated with control medium, $2 \mu \mathrm{M}$ of Gefitinib (GEF) or $5 \mu \mathrm{M}$ of Erlotinib (ERLO) and exposed to CSE (0 or 1\%) for $24 \mathrm{~h}$. Total lysates were prepared in the presence of protease and phosphatase inhibitors [26, 28, 34]. Lysates were analyzed by immunoblotting with an antibody to paxillin or phospho-paxillin. Protein levels were normalized by immunoblotting with an antibody against betaactin. Vertical lines separate independent experiments. (B). J82 bladder cancer cells were transfected with the control (scrambled) siRNA, or siRNA against PIG-U, PIG-T and GPAA1 for $72 \mathrm{~h}$ and then exposed to control medium (0) or 1\% CSE for an additional 24 h. Levels of paxillin (PAX), phospho-paxillin (p-PAX), PIG-U, PIG-T or GPAA1 were tested by immunoblotting with antibodies described in Materials and Methodology section. Protein levels were normalized by immunoblotting with an antibody against betaactin. Vertical lines separate independent experiments. 


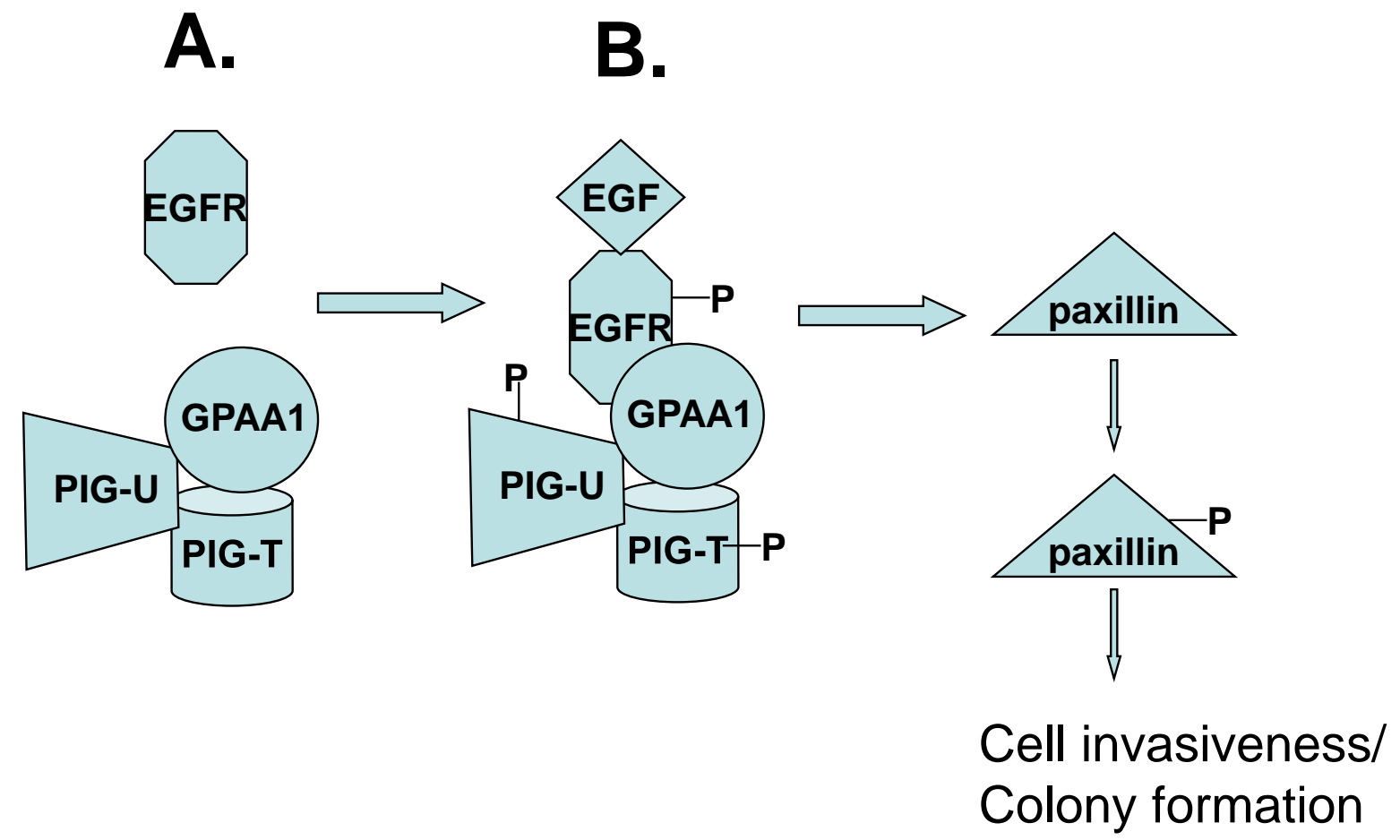

Fig. (10). Schematic representation of PIG-U/PIG-T/GPAA1 protein interactions with EGFR affecting their tyrosine phosphorylation upon exposure of cancer cells to EGF or CSE (Panel B). Panel A depicts the situation without exposure.

\section{DISCUSSION}

Glycoconjugates are utilized by eukaryotic organisms ranging from yeast to humans for the cell surface expression of a wide variety of proteins and lipids that expressed as enzymes or receptors and serve a diversity of functions, including cell signaling and cell survival $[6,29,31,32]$. Glycosylphosphatidylinositols (GPIs) represent a universal structure for the anchorage of glycoconjugated substrates to cellular membranes [6, 29, 31, 32].

We previously found that the protein subunits of GPItransamidase complex (e.g. PIG-U, PIG-T and GPAA1) play critical roles in human bladder and breast cancers functioning as oncogenes and manifesting their tumorigenic activities through signaling mechanisms that involve urokinase receptor/Stat3 pathway and paxillin phosphorylation pathway [2628]. We also showed that GPAA1 is overexpressed in HNSCC cells as well [35]. We predicted that the physiologic and pathophysiologic functions of these proteins manifested through their interaction with other cellular components, including protein kinases and substrates. Since, these proteins are known to form complexes to generate GPItransamidase activity necessary for membranal targeting of certain proteins, it was plausible to ascertain the role of PIGU/PIG-T/GPAA1 protein associations in human cancers.

We therefore found that PIG-U, PIG-T and GPAA1 proteins formed complexes between each other and EGFR in HNSCC, bladder and breast cancer cells upon EGF exposure. We also found that while GPAA1 directly associated with EGFR, PIG-U and PIG-T served as substrates for EGFR tyrosine kinase, a well-known oncogenic signaling molecule [1]. We observed that inhibitors of EGFR tyrosine kinase, Gefitinib or Erlotinib, modulated a tyrosine phosphorylation of PIG-U and PIG-T, while failed to affect pro- tein association between EGFR and GPAA1 induced by EGF. We also showed that CSE induced colony formation and invasiveness of human bladder cancer cells in vitro. CSE enhanced the protein association between PIG-U, PIG-T and GPAA1 and between GPAA1 and EGFR in human cancers. CSE also increased the levels of PIG-T and PIG-U phosphorylated at the tyrosine residues in human bladder and breast cancer cells. We then found that EGFR tyrosine kinase inhibitors and siRNA silencing of PIG-U, PIG-T or GPAA1 led to modulation of CSE-induced increase in colony formation and invasiveness of human bladder cancer cells. We thus suggested a novel mechanism overlapping the oncogenic potential of PIG-U, PIG-T and GPAA1 that implicates EGFR tyrosine phosphorylation of PIG-U or PIG-T, which were tethered to EGFR by protein-protein interaction with GPAA1, in turn leading to paxillin phosphorylation (Fig. 10). The further studies examining the cross talk between GPI-anchoring signaling pathway and EGFR signaling pathway would provide us with additional opportunities in developing novel therapeutics for human epithelial cancers.

\section{CONCLUSION}

We propose a novel mechanism overlapping the oncogenic potential of PIG-U, PIG-T and GPAA1 that implicates EGFR tyrosine phosphorylation of PIG-U or PIG-T and subsequent paxillin phosphorylation upon exposure of epithelial tumor cells (e.g. bladder, breast and head and neck cancers) to smoking.
ABBREVIATIONS
SHS
$=$ second-hand smoking
$\mathrm{CSE}=$ cigarette smoking extract
GPI = glycosylphosphatidyl inositol 


$$
\begin{aligned}
& \text { EGF }=\text { epidermal growth factor } \\
& \text { EGFR }=\text { EGF receptor } \\
& \text { HNSCC }=\text { head and neck squamous cell carcinoma } \\
& \text { ATCC }=\text { American Tissue Culture Collection } \\
& \text { HEK }=\text { human embryonic kidney cells } \\
& \text { DMSO }=\text { demethylsulfoxide } \\
& \text { BD }=\text { binding domain } \\
& \text { AD }=\text { activation domain } \\
& \text { FBS }=\text { fetal bovine serum } \\
& \text { siRNA }=\text { small interference RNA. }
\end{aligned}
$$

\section{ACKNOWLEDGEMENTS}

This work was supported in part by the grants from the Flight Attendant Medical Research Institution (for B.T and E.R.).

\section{REFERENCES}

[1] Williams MD, Sandler AB. The epidemiology of lung cancer. Cancer Treat Res 2001; 105: 31-52.

[2] Asomaning K, Miller DP, Liu G, et al. Second hand smoke, age of exposure and lung cancer risk. Lung Cancer 2008; 61:13-20.

[3] Boffetta P. Human cancer from environmental pollutants: the epidemiological evidence. Mutat Res 2006; 608: 157-62.

[4] Lesmes GR, Donofrio KH. Passive smoking: the medical and economic issues. Am J Med 1992; 93: 38S-42S.

[5] Lin Y, Kikuchi S, Tamakoshi K, et al. Active smoking, passive smoking, and breast cancer risk: findings from the Japan Collaborative Cohort Study for Evaluation of Cancer Risk. J Epidemiol 2008; 18: 77-83.

[6] Ong EK, Glantz SA. Tobacco industry efforts subverting International Agency for Research on Cancer's second-hand smoke study. Lancet 2000; 355: 1253-59.

[7] Wilson N, Thomson G. Still dying from second-hand smoke at work: a brief review of the evidence for smoke-free workplaces in New Zealand. N Z Med J 2002; 115: U240.

[8] Garces YI, Schroeder DR, Nirelli LM, et al. Second primary tumors following tobacco dependence treatments among head and neck cancer patients. Am J Clin Oncol 2007; 30: 531-9.

[9] Hecht SS. Human urinary carcinogen metabolites: biomarkers for investigating tobacco and cancer. Carcinogenesis 2002; 23: 907-22.

[10] Jiang X, Yuan JM, Skipper PL, Tannenbaum SR, Yu MC. Environmental tobacco smoke and bladder cancer risk in never smokers of Los Angeles County. Cancer Res 2007; 67: 7540-5.

[11] La Rocca G, Anzalone R, Magno F, Farina F, Cappello F, Zummo G. Cigarette smoke exposure inhibits extracellular MMP-2 (gelatinase A) activity in human lung fibroblasts. Respir Res 2007; 8: 23-9.

[12] Liu H, Zhou Y, Boggs SE, Belinsky SA, Liu J. Cigarette smoke induces demethylation of prometastatic oncogene synucleingamma in lung cancer cells by downregulation of DNMT3B. Oncogene 2007; 26: 5900-10.

[13] Lubin JH, Alavanja MC, Caporaso N, et al. Cigarette smoking and cancer risk: modeling total exposure and intensity. Am J Epidemiol 2007; 166: 479-89.

[14] Marsit CJ, Karagas MR, Schned A, Kelsey KT. Carcinogen exposure and epigenetic silencing in bladder cancer. Ann N Y Acad Sci 2006; 1076: 810-21.

[15] Marsit CJ, Houseman EA, Schned AR, Karagas MR, Kelsey KT. Promoter hypermethylation is associated with current smoking, age, gender and survival in bladder cancer. Carcinogenesis 2007; 28: 1745-51.

[16] Mercer BA, Kolesnikova N, Sonett J, D'Armiento J. Extracellular regulated kinase/mitogen activated protein kinase is up-regulated in pulmonary emphysema and mediates matrix metalloproteinase-1 induction by cigarette smoke. J Biol Chem 2004; 279: 17690-6.
Mohseni M, Zand S, Aghamir S. Effect of smoking on prognostic factors of transitional cell carcinoma of the bladder. Urol J 2004; 1 : 250-2.

Moore LE, Pfeiffer RM, Poscablo C, et al. Genomic DNA hypomethylation as a biomarker for bladder cancer susceptibility in the Spanish Bladder Cancer Study: a case-control study. Lancet Oncol 2008; 9: 359-66.

Shankar A, Yuan JM, Koh WP, Lee HP, Yu MC. Morbidity and mortality in relation to smoking among women and men of Chinese ethnicity: the Singapore Chinese Health Study. Eur J Cancer 2008; 44: 100-9.

Shen YJ, Ye DW, Yao XD, et al. Overexpression of CDC91L1 (PIG-U) in bladder urothelial cell carcinoma: correlation with clinical variables and prognostic significance. BJU Int 2008; 101: 1139.

Siemiatycki J, Dewar R, Krewski D, Désy M, Richardson L, Franco E. Are the apparent effects of cigarette smoking on lung and bladder cancers due to uncontrolled confounding by occupational exposures? Epidemiology 1994; 5: 57-65.

[22] Zhou W, Heist RS, Liu G, et al. Second hand smoke exposure and survival in early-stage non-small-cell lung cancer patients. Clin Cancer Res 2006; 12: 7187-93.

[23] Husgafvel-Pursiainen K. Genotoxicity of environmental tobacco smoke: a review. Mutat Res 2004; 567: 427-45.

[24] Wu X, Gu J, Grossman HB, et al. Bladder cancer predisposition: a multigenic approach to DNA-repair and cell-cycle-control genes. Am J Hum Genet 2006; 78: 464-79.

[25] Zhou Y, Gu X, Ashayeri E, Zhang R, Sridhar R. Nicotine decreases the cytotoxicity of doxorubicin towards MCF-7 and KB-3.1 human cancer cells in culture. J Natl Med Assoc 2007; 99: 319-27.

[26] Guo Z, Linn JF, Wu G, et al. CDC91L1 (PIG-U) is a newly discovered oncogene in human bladder cancer. Nat Med 2004; 10 374-81.

[27] Nagpal JK, Dasgupta S, Jadallah S, et al. Profiling the expression pattern of GPI transamidase complex subunits in human cancer. Mod Pathol 2008; 21: 979-91.

[28] Wu G, Guo Z, Chatterjee A, et al. Overexpression of glycosylphosphatidylinositol (GPI) transamidase subunits phosphatidylinositol glycan class $\mathrm{T}$ and/or GPI anchor attachment 1 induces tumorigenesis and contributes to invasion in human breast cancer. Cancer Res 2006; 66: 9829-36.

[29] Hong Y, Ohishi K, Kang JY, et al. Human PIG-U and yeast Cdc91p are the fifth subunit of GPI transamidase that attaches GPIanchors to proteins. Mol Biol Cell 2003; 14: 1780-9.

[30] Ohishi K, Inoue N, Kinoshita T. PIG-S and PIG-T, essential for GPI anchor attachment to proteins, form a complex with GAA1 and GPI8. EMBO J 2001; 20: 4088-98.

[31] Vainauskas S, Maeda Y, Kurniawan H, Kinoshita T, Menon AK. Structural requirements for the recruitment of Gaal into a functional glycosylphosphatidylinositol transamidase complex. J Biol Chem 2002; 277: 30535-42.

[32] Vainauskas S, Menon AK. Endoplasmic reticulum localization of Gaa1 and PIG-T, subunits of the glycosylphosphatidylinositol transamidase complex. J Biol Chem 2005; 280: 16402-9.

[33] Carp H, Janoff A. Possible mechanisms of emphysema in smokers: in vitro suppression of serum elastase-inhibitory capacity by fresh cigarette smoke and its prevention by antioxidants. Am Rev Respir Dis 1978; 118: 617-21.

[34] Huang Y, Hoque MO, Wu F, Trink B, Sidransky D, Ratovitski E Midkine induces epithelial-mesenchymal transition through the Notch2-Jak2-Stat3 signaling in human keratinocytes. Cell Cycle 2008; 7: 1613-22.

[35] Jiang WW, Zahurak M, Zhou ZT, et al. Alterations of GPI transamidase subunits in head and neck squamous carcinoma. Mol Cancer 2007; 21: 74-9.

[36] Canel M, Secades P, Garzón-Arango M, et al. Involvement of focal adhesion kinase in cellular invasion of head and neck squamous cell carcinomas via regulation of MMP-2 expression. Br J Cancer 2008; 98: 1274-84

[37] Conway WC, Van der Voort van Zyp J, Thamilselvan V, Walsh MF, Crowe DL, Basson MD. Paxillin modulates squamous cancer cell adhesion and is important in pressure-augmented adhesion. $\mathrm{J}$ Cell Biochem 2006; 98: 1507-16.

[38] Huang C, Jacobson K, Schaller MD. A Role for JNK-Paxillin signaling in cell migration. Cell Cycle 2004; 3: 4-6. 
[39] Ishibe S, Joly D, Liu Z.X, Cantley L.G. Paxillin serves as an ERKregulated scaffold for coordinating FAK and Rac activation in epithelial morphogenesis. Mol Cell 2004; 16: 257-67.

[40] Ridky T, Khavari PA. Pathways sufficient to induce epidermal carcinogenesis. Cell Cycle 2004; 3: 621-4.

[41] Schaller M. FAK and paxillin: regulators of N-cadherin adhesion and inhibitors of cell migration? J Cell Biol 2004; 166: 157-9.
[42] Zaidel-Bar R, Milo R, Kam Z, Geiger B. A paxillin tyrosine phosphorylation switch regulates the assembly and form of cell-matrix adhesions. J Cell Sci 2007; 120: 137-48.

[43] Andl CD, Mizushima T, Oyama K, Bowser M, Nakagawa H, Rustgi AK. EGFR-induced cell migration is mediated predominantly by the JAK-STAT pathway in primary esophageal keratinocytes. Am J Physiol Gastrointest Liver Physiol 2004; 287: G122737.

Received: December 17, 2008

(C) Huang et al.; Licensee Bentham Open.

This is an open access article licensed under the terms of the Creative Commons Attribution Non-Commercial License (http://creativecommons.org/licenses/by-nc/3.0/) which permits unrestricted, non-commercial use, distribution and reproduction in any medium, provided the work is properly cited. 